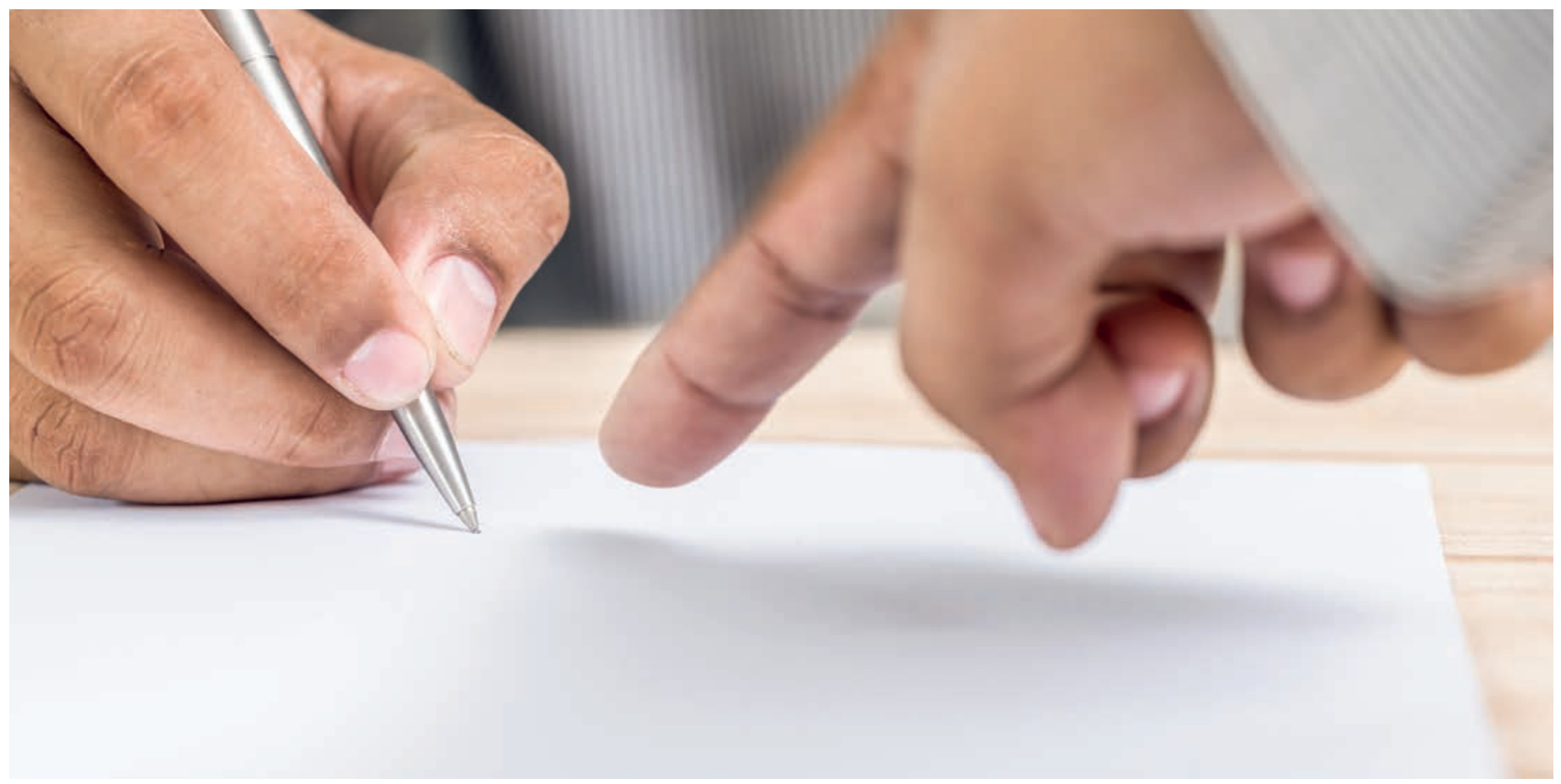

\title{
Arbeitszeugnis - Arztgewissen auf der Anklagebank
}

\section{Jürg Kesselring}

Prof. Dr. med., FRCP, Senior Botschafter und Neuroexperte, ehem. Chefarzt Neurologie und Neurorehabilitation, Rehabilitationszentrum Valens

Es kann ja vorkommen, dass ein Assistenzarzt (wohl auch eine Ärztin) mit einem ausländischen Arztdiplom in eine Klinik gelangt und dort mit einem Arbeitsvertrag angestellt wird zu den üblichen Arbeitsbedingungen in prächtiger Umgebung mit spannenden Aufgaben, gutem Lohn, angemessenen (= reichlich) Ferien- und Kompensationstagen sowie systematischer fachlicher Fort- und Weiterbildung. Eine solche Anstellung stützt sich wohl auch zum Teil auf Arbeitszeugnisse früherer Arbeitgeber und mündliche Referenzen.

\section{Mitarbeiter mit Makeln}

Nun mag es sein, dass sich dieser Mitarbeiter nicht ganz so sehr bewährt, wie man sich dies als verantwortlicher Chefarzt wünscht: Er ist sehr oft - freilich mit entsprechenden Zeugnissen von Psychiatern, All- gemein- und Spezialärzten - krankheitsbedingt abwesend. Die Kolleginnen und Kollegen springen ein, die anfallenden Aufgaben zu erledigen. Die sprachlichen Einschränkungen ermöglichen kaum direkten Kontakt mit den Patientinnen und Patienten, die Arztbriefe werden von Oberärzten regelmässig aufwendig

Das Arbeitszeugnis fällt etwas diskret, aber anständig formuliert aus, mit guten Wünschen für den weiteren Lebensweg.

korrigiert oder neu geschrieben, zu den Besprechungen und Rapporten kommt er mit einer für uns eine Spur befremdlichen Konsequenz immer (= immer!) zu spät, ein Interesse am fachlichen Austausch mit Kollegen oder Vorgesetzten oder persönliche Fürsorge für Patientinnen und Mitarbeiter lässt sich am Verhalten nicht ablesen, der fachliche Input tendiert gegen null. 
Das Arbeitszeugnis nach einem Jahr der Versuche und des Ausharrens fällt etwas diskret, aber anständig formuliert aus mit guten Wünschen für den weiteren Lebensweg.

\section{OR-Artikel 328}

Nun kommt aber vier Wochen nach Ablauf des Arbeitsverhältnisses ein eingeschriebener Brief mit dem Vermerk "persönlich» an den Chefarzt, in dem ein Rechtsanwalt (es könnte bestimmt auch einmal eine Anwältin sein) diesen auf OR-Artikel 328 hinweist (andere Fachexperten bemühen in diesem Zusammenhang eher OR 330a), wonach «der Arbeitgeber die Persönlichkeit des Arbeitnehmers zu achten und $\mathrm{zu}$ schützen und auf dessen Gesundheit gebührende Rücksicht zu nehmen und für die Wahrung der Sittlichkeit zu sorgen» habe etc. Das dürfte ja wohl jedem

\section{Ein Arbeitszeugnis muss eigentlich laut Recht- sprechung nicht nur wohlwollend, schriftlich, vollständig usw. sein, sondern auch wahr.}

verantwortungsbewussten Arzt klar sein und einleuchten. Es folgen dann konkrete Drohungen: «Kommt der Arbeitgeber dieser Pflicht nicht nach, kann er zur Zahlung einer Entschädigung bis zu sechs Monatslöhnen und einer Genugtuung verpflichtet werden.» Dann wird konkret vorgeschrieben, wie der Wortlaut des Arbeitszeugnisses abzuändern sei: So müsste also geschrieben werden, dass der Kollege «bereits gute medizinische Kenntnisse und Berufserfahrungen mitgebracht habe», dass es ihm "gelungen» sei, «sich schnell in das Fachgebiet ... aber auch in die Nachbarbereiche ... einzuarbeiten ... nach kurzer Zeit konnte er selbstständig klinische ... Status in deutscher Sprache erstellen ... fiel ihm die Beurteilung ... Notfälle sowie Anpassung der Medikation leicht ... War ein zuverlässiger, belastbarer, pflichtbewusster sowie interessierter Mitarbeiter ... handelte patientenorientiert, selbstständig, effizient und organisiert ... Anforderungen erfüllte er jederzeit vollumfänglich ...", und dann kommt noch der Befehl zur sprachlichen Unmöglichkeit: Er habe «zur vollsten Zufriedenheit gearbeitet» - voll ist voll und nicht zu steigern (vgl. Wasserglas). Von Rechtsanwaltsseite wurde der Angeschriebene «des Weiteren darauf hingewiesen, dass mündliche Referenzen nicht vom Arbeitszeugnis abweichen dürfen».

\section{Ein Arbeitszeugnis nach den Wünschen des ehemaligen Mitarbeiters?}

Soll nun diesen Forderungen Genüge getan und das Zeugnis entsprechend geändert werden? Es ist ja wohl nicht unproblematisch, ein Zeugnis nach den Wünschen ehemaliger Angestellter zu verfassen. Es muss eigentlich laut Rechtsprechung nicht nur wohlwollend, schriftlich, vollständig usw. sein, sondern auch wahr. Wenn es also zugunsten ehemaliger Mitarbeiter ausgestellt wird und nicht mehr der Wahrheit entspricht, kann ein zukünftiger Arbeitgeber darauf zurückkommen. Man (= ich) könnte also auch schadenersatzpflichtig werden, falls ein zukünftiger Arbeitgeber zu einem Schaden kommt, weil er den Arzt wegen des falschen oder zumindest einseitigen Arbeitszeugnisses eingestellt hat. Es kann problematisch sein, Änderungen entgegen dem eigenen Gewissen vorzunehmen, auch wenn sie unter Druck erfolgen.

Den geplanten Zusatz im Begleitbrief, den ich gerne geschrieben hätte, konnte ich mir gerade noch verkneifen: "Als gewissenhafter Arzt bedauere ich, dass Sie mich unter erheblichen Drohungen dazu nötigen, die Unwahrheit zu sagen und mit meiner Unterschrift zu beglaubigen. Als Schweizer Bürger erstaunt es mich, dass Sie mir auch vorschreiben, was ich im Umgang mit ärztlichen Kollegen sagen darf und was nicht. Wir erfreuen uns doch sonst in der Schweiz einer gewissen Redefreiheit.» Bei Anfragen kann ja dann keine Aussage auch eine Aussage sein.

Bildnachweis

(c) Witthaya Prasongsin | Dreamstime.com 